\title{
Analysis of Bitcoin Returns Volatility using AR-GARCH Modelling
}

\author{
Mihir Dash \\ Alliance University \\ Bangalore, India
}

\begin{abstract}
Bitcoin is a virtual/cryptocurrency, serving as a decentralized medium of digital exchange and not tied to any financial institution. It gained in popularity in the aftermath of the global financial crisis with the failure of many prominent banks and financial institutions, as it provided investors with direct control over their money.

The supply of Bitcoins is constrained due to its geometrically decreasing growth rate, with a limiting maximum supply of twenty-one million Bitcoins. Because of the limited number of Bitcoins in circulation and their increasing demand, Bitcoin prices tend to be highly volatile and increase/decrease at a very fast pace. Many noted economists have characterised Bitcoin prices as a speculative bubble. However, it is expected that, with wider acceptance and adoption of Bitcoins, Bitcoin prices would settle down and its volatility would stabilise.

The study examines the stability of Bitcoin price/returns volatility using an AR-GARCH model. The data for the study were the daily closing Bitcoin prices obtained from the bitcoin,com website ${ }^{1}$ for the study period 01/01/2013 - 31/12/2017.
\end{abstract}

Keywords: Bitcons, crypto-currency, volatility, speculative bubble, AR-GARCH model, stability.

\section{Introduction}

Bitcoin is the world's first digital cryptocurrency. It was first released as an open source by Satoshi Nakamoto in 2008. Bitcoins serve as a decentralised medium of digital exchange and are not tied to any financial institution. When large banking federations lost control over their management policies, investors shifted to cryptocurrencies to retain control over their money.

Bitcoins are not physically formed at all. They are mined through a process of transaction verification and solving a computational puzzle. The miner, a special user authorised to verify and secure the transactions, who solves the puzzle first gets the opportunity to claim Bitcoins as a reward, which becomes the newly released currency.

Bitcoin transactions are made through a public key as the address (similar to a bank account number). All the transaction details of any Bitcoin address are verified and recorded in a public distributed ledger (the blockchain) without the need for a trusted recordkeeping authority or central intermediary. Transaction blocks contain an SHA-256 cryptographic hash of previous transaction blocks, and are chained together, serving as an immutable record of all transactions that have ever occurred.

\footnotetext{
${ }^{1}$ https://charts.bitcoin.com/chart/price
} 


\section{Problem Identification}

At the end of the last quarter of 2017, there were 16,794,762 Bitcoins in circulation, and 876,989 Bitcoin users (unique addresses); since Bitcoin's inception in 2009, there had been a total of $291,342,443$ Bitcoin transactions, representing about $66.8 \%$ of the total estimated crypto-currency capitalisation. The number of bitcoins generated per block is set to decrease geometrically, with a $50 \%$ reduction every 210,000 blocks, or approximately four years. As a result, the limiting maximum supply of Bitcoins is twenty-one million.

Because of the limited number of Bitcoins in circulation, and the increased demand with each passing day, Bitcoin is highly volatile and increases or decreases at a very fast pace. Chu et al (2017) suggested that cryptocurrencies are highly volatile compared to traditional currencies. Cheah and Fry (2015) suggested that the Bitcoin market is highly speculative as compared to other currencies.

Speculators wish to take advantage of the volatility, but genuine investors think of it as risky; so, many investors do not invest in Bitcoins. Many noted economists, including Nobel laureates Paul Krugman, Robert J. Shiller, Joseph Stiglitz, Richard Thaler, James Heckman, Thomas Sargent, Angus Deaton, and Oliver Hart, have characterised Bitcoin prices as a speculative bubble. However, it is expected that, with wider acceptance and adoption, Bitcoin prices would settle down and its volatility would stabilise.

\section{Literature Review}

Several studies have used econometric modelling, specifically GARCH-type models, to analyse Bitcoin prices (Zheng, 2018). Bouoiyour and Selmi (2016) examined Bitcoin volatility using the CMT-GARCH model, which they found to be optimal in terms of AIC, BIC, and HQ criteria. They suggested that Bitcoin volatility had reduced considerably from Jan'15. They also found evidence of asymmetric reaction to negative shocks as against positive shocks. Stavroyiannis and Babalos (2017) examined the dynamic properties of Bitcoin in the US market using univariate and multivariate GARCH models and Vector Auto-Regressive models. They found no evidence to suggest that Bitcoins provide investors with the benefits of hedge, diversifier, or safe-haven with respect to the movement of the US stock markets, in contrast to gold, which is used by investors in order to limit their losses, especially during adverse market conditions. Katsiampa (2017) compared different GARCH models for Bitcoin volatility estimation. He found that the optimal conditional heteroskedasticity model (in the sense of AIC, BIC, and Log-Likelihood criteria) is the AR-CGARCH model, highlighting the significance of including both a short-run and a longrun component of the conditional variance. Chu et al (2017) compared GARCH-type models for the seven most popular cryptocurrencies, viz. Bitcoin, Dash, Dogecoin, Litecoin, Maidsafecoin, Monero, and Ripple. They found that the $\operatorname{IGARCH}(1,1)$ model gives the best fit for Bitcoin, Dash, Litecoin, Maidsafecoin and Monero; the GJRGARCH $(1,1)$ model gives the best fit for Dogecoin; and the GARCH $(1,1)$ model gives the best fit for Ripple.

The present study contributes to the literature by attempting to quantify the variation of Bitcoin returns using the AR-GARCH models. 


\section{Methodology}

The objective of the study is to understand and model the volatility of Bitcoin returns using univariate time series models.

The data for the study were the daily closing Bitcoin prices obtained from the bitcoin,com website ${ }^{2}$ for the study period 01/01/2013 - 31/12/2017. Following Bouoiyour and Selmi (2016), the Bitcoin returns series was analysed in the subperiod 01/01/2015 - 31/12/2017 separately and compared with the overall period. The Bitcoin returns series were computed from the closing prices using the log-returns formula

$$
r_{t}=\ln \left(B_{t} / B_{t-1}\right)
$$

In order to tests for stationarity of the Bitcoin returns series, the ADF unit root test was applied. The specification of the ADF unit root test used in the study is given by

$$
\Delta r_{t}=\alpha+\beta t+\gamma r_{t-1}+\delta_{1} \Delta r_{t-1}+\cdots+\delta_{k} \Delta r_{t-k}+\epsilon_{t}
$$

i.e. with trend and lagged first order differences of the log-returns. The lag structure was selected using the Akaike Information Criterion (AIC) and/or the Bayesian Information Criterion (BIC). The null hypothesis of a unit root is rejected if the coefficient $\gamma$ is statistically significant and negative.

In order to understand the autocorrelation structure of the Bitcoin returns series, the ACF and PACF correlograms were examined. The Bitcoin returns series was further modelled using the ARMA model. The specification of the $\operatorname{ARMA}(p, q)$ model used in the study is given by

$$
r_{t}=a+\phi_{1} r_{t-1}+\cdots+\phi_{p} r_{t-p}+\epsilon_{t}+\theta_{1} \epsilon_{t-1}+\cdots+\theta_{q} r_{t-q}
$$

The AR and MA lags $\mathrm{p}$ and $\mathrm{q}$ were selected to minimise the AIC and BIC. The model was estimated using the maximum likelihood method.

In order to understand the volatility dynamics of the Bitcoin returns series, the AR-GARCH model was used. The specification of the $\operatorname{AR}(p)-\operatorname{GARCH}(1,1)$ model used in the study is as follows. The conditional mean equation is given by

$$
r_{t}=\mu+\varphi_{1} r_{t-1}+\cdots+\varphi_{p} r_{t-p}+\epsilon_{t}
$$

with $\varepsilon_{t}=\sigma_{t} h_{t}$, where $\left\{h_{t}\right\}$ is a strong white noise process, and the conditional variance equation is given by

$$
\sigma_{t}^{2}=\omega+\alpha_{1} \epsilon_{t-1}^{2}+\beta_{1} \sigma_{t-1}^{2}
$$

The AR lag length $\mathrm{p}$ was selected to minimise the AIC and BIC. The model was estimated using the maximum likelihood method.

\footnotetext{
${ }^{2}$ https://charts.bitcoin.com/chart/price
} 


\section{Findings}

The results of the ADF tests are presented in the tables below.

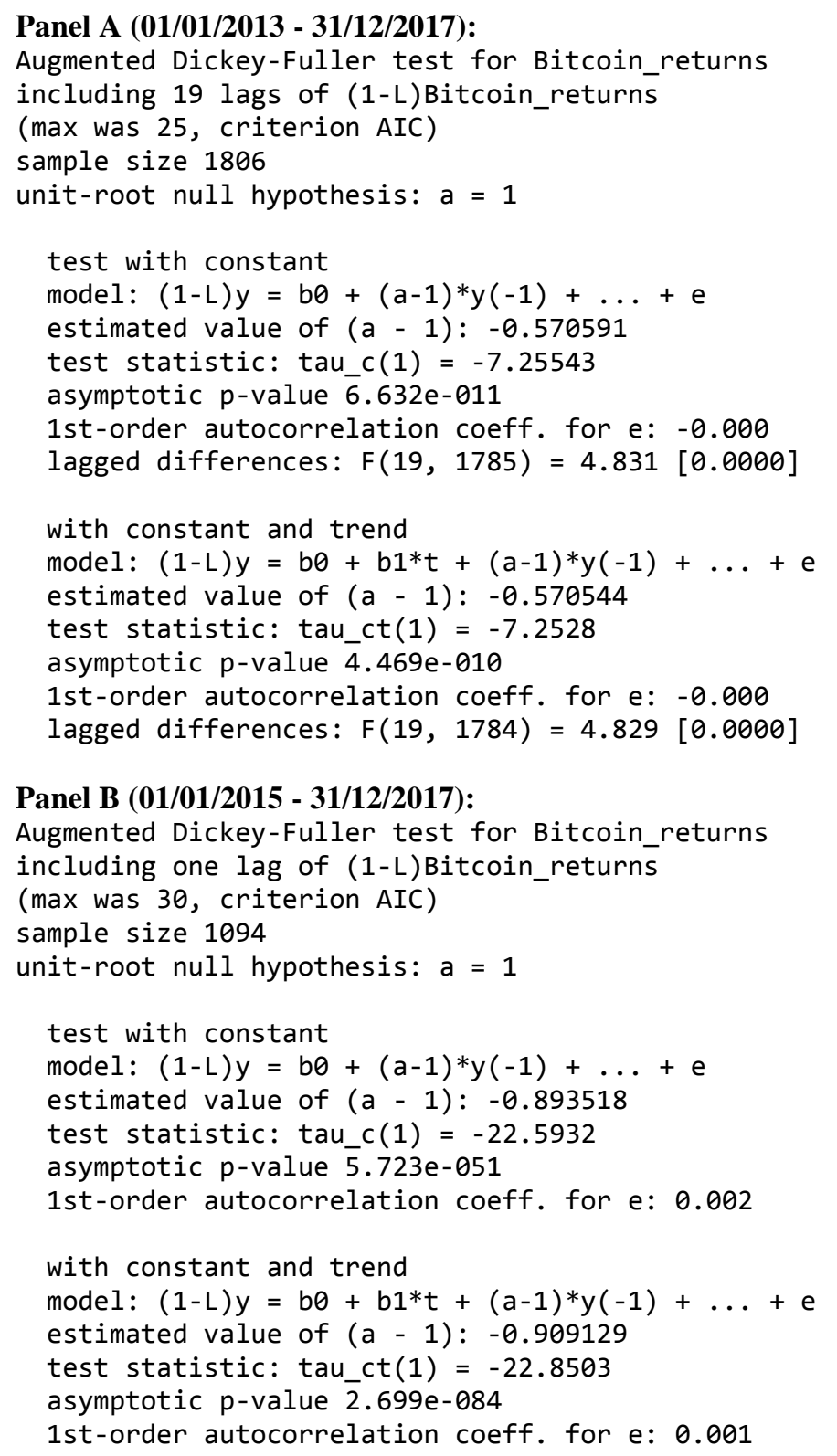

The results of the ADF tests indicate that the null hypothesis of unit root was rejected for Bitcoin returns in both panels. This implies stationarity of the Bitcoin returns series. 
The ACF and PACF correlograms for Bitcoin returns are presented below.

Panel A (01/01/2013 - 31/12/2017):

Autocorrelation function for Bitcoin_returns $* * *, * *, *$ indicate significance at the $1 \%, 5 \%, 10 \%$ levels using standard error $1 / T^{\wedge} 0.5$

\begin{tabular}{|c|c|c|c|c|c|c|}
\hline LAG & $\mathrm{ACF}$ & & PACF & & Q-stat. & [p-value] \\
\hline 1 & 0.1767 & $* * *$ & 0.1767 & $* * *$ & 57.1230 & {$[0.000]$} \\
\hline 2 & -0.0051 & & -0.0375 & & 57.1700 & {$[0.000]$} \\
\hline 3 & -0.0227 & & -0.0156 & & 58.1110 & {$[0.000]$} \\
\hline 4 & 0.0433 & $*$ & 0.0517 & $* *$ & 61.5491 & {$[0.000]$} \\
\hline 5 & 0.1551 & $* * *$ & 0.1421 & $* * *$ & 105.6306 & {$[0.000]$} \\
\hline 6 & 0.0329 & & -0.0197 & & 107.6104 & {$[0.000]$} \\
\hline 7 & -0.0516 & $* *$ & -0.0501 & $* *$ & 112.5026 & {$[0.000]$} \\
\hline 8 & -0.0788 & $* * *$ & -0.0581 & $* *$ & 123.8899 & {$[0.000]$} \\
\hline 9 & -0.0159 & & -0.0034 & & 124.3539 & [0.000] \\
\hline 10 & 0.0991 & $* * *$ & 0.0814 & $* * *$ & 142.3988 & {$[0.000]$} \\
\hline 11 & 0.0675 & $* * *$ & 0.0370 & & 150.7857 & {$[0.000]$} \\
\hline 12 & 0.0051 & & 0.0073 & & 150.8333 & {$[0.000]$} \\
\hline 13 & -0.0196 & & 0.0026 & & 151.5421 & {$[0.000]$} \\
\hline 14 & 0.0284 & & 0.0317 & & 153.0234 & {$[0.000]$} \\
\hline 15 & 0.0734 & $* * *$ & 0.0314 & & 162.9456 & {$[0.000]$} \\
\hline 16 & 0.0585 & $* *$ & 0.0210 & & 169.2615 & {$[0.000]$} \\
\hline 17 & 0.0680 & $* * *$ & 0.0651 & $* * *$ & 177.7829 & {$[0.000]$} \\
\hline 18 & -0.0242 & & -0.0304 & & 178.8675 & {$[0.000]$} \\
\hline 19 & 0.0155 & & 0.0267 & & 179.3127 & {$[0.000]$} \\
\hline 20 & 0.0706 & $* * *$ & 0.0465 & $* *$ & 188.5265 & {$[0.000]$} \\
\hline 21 & 0.0460 & $* *$ & 0.0068 & & 192.4460 & {$[0.000]$} \\
\hline 22 & 0.0062 & & -0.0138 & & 192.5171 & {$[0.000]$} \\
\hline 23 & -0.0658 & $* * *$ & -0.0495 & $* *$ & 200.5267 & {$[0.000]$} \\
\hline 24 & -0.0350 & & -0.0167 & & 202.8017 & {$[0.000]$} \\
\hline 25 & 0.0054 & & -0.0077 & & 202.8550 & {$[0.000]$} \\
\hline 26 & 0.0263 & & 0.0102 & & 204.1367 & {$[0.000]$} \\
\hline 27 & 0.0388 & $*$ & 0.0318 & & 206.9315 & {$[0.000]$} \\
\hline 28 & -0.0240 & & -0.0126 & & 208.0002 & {$[0.000]$} \\
\hline 29 & -0.0224 & & -0.0078 & & 208.9317 & {$[0.000]$} \\
\hline 30 & -0.0059 & & -0.0203 & & 208.9967 & {$[0.000]$} \\
\hline
\end{tabular}


ACF for Bitcoinreturns

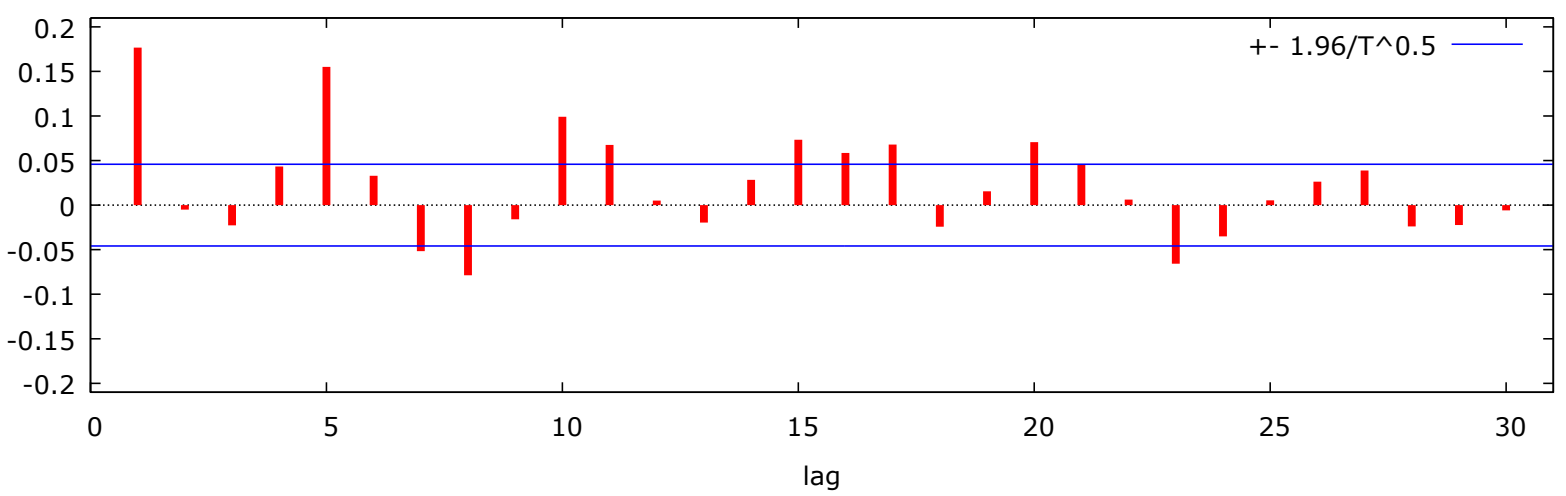

PACF for Bitcoinreturns

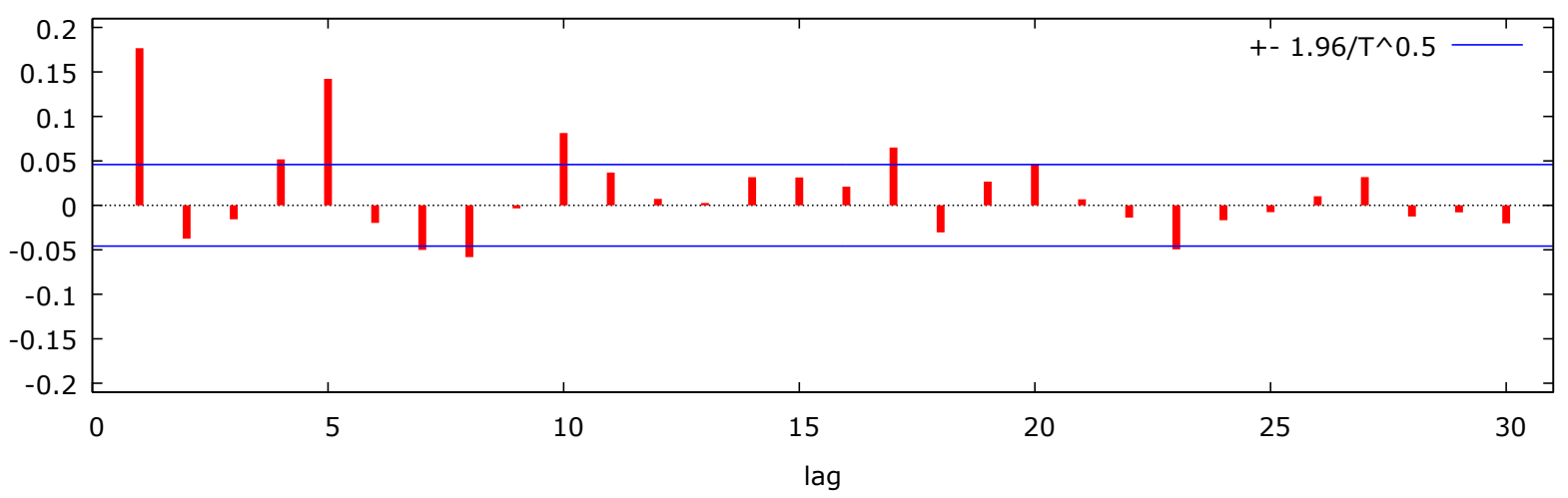

Panel B (01/01/2015 - 31/12/2017):

Autocorrelation function for Bitcoin_returns $* * *, * *, *$ indicate significance at the $1 \%, 5 \%, 10 \%$ levels using standard error $1 / \mathrm{T}^{\wedge} 0.5$

$\begin{array}{rrlrlll}\text { LAG } & \text { ACF } & & \text { PACF } & \text { Q-stat. } & {[\text { p-value }]} \\ 1 & 0.1553 & * * * & 0.1553 * * * & 26.4963 & {[0.000]} \\ 2 & -0.0342 & & -0.0598 * * & 27.7831 & {[0.000]} \\ 3 & 0.0100 & & 0.0256 & 27.8939 & {[0.000]} \\ 4 & -0.0084 & & -0.0167 & 27.9721 & {[0.000]} \\ 5 & 0.0592 & * * & 0.0668 & * * & 31.8410 & {[0.000]} \\ 6 & 0.0179 & & -0.0044 & 32.1945 & {[0.000]} \\ 7 & -0.0395 & & -0.0361 & 33.9158 & {[0.000]} \\ 8 & -0.0228 & & -0.0125 & 34.4899 & {[0.000]} \\ 9 & 0.0322 & & 0.0371 & 35.6374 & {[0.000]} \\ 10 & 0.0504 & * & 0.0366 & 38.4476 & {[0.000]} \\ 11 & 0.0355 & & 0.0236 & 39.8418 & {[0.000]} \\ 12 & -0.0053 & & -0.0086 & 39.8730 & {[0.000]} \\ 13 & -0.0240 & & -0.0171 & 40.5120 & {[0.000]} \\ 14 & 0.0361 & & 0.0390 & 41.9654 & {[0.000]} \\ 15 & 0.0093 & & -0.0110 & 42.0622 & {[0.000]} \\ 16 & -0.0004 & & 0.0021 & 42.0623 & {[0.000]} \\ 17 & 0.0148 & & 0.0171 & 42.3060 & {[0.001]} \\ 18 & 0.0458 & & 0.0494 & 44.6440 & {[0.000]} \\ 19 & 0.0447 & & 0.0259 & 46.8777 & {[0.000]} \\ 20 & 0.0079 & & -0.0061 & 46.9482 & {[0.001]} \\ 21 & -0.0584 & * & -0.0594 & * * & 50.7633 & {[0.000]}\end{array}$




\begin{tabular}{|c|c|c|c|c|c|c|}
\hline 22 & -0.0014 & & 0.0197 & & 50.7654 & {$[0.000]$} \\
\hline 23 & -0.0353 & & -0.0502 & $*$ & 52.1598 & {$[0.000]$} \\
\hline 24 & -0.0152 & & -0.0037 & & 52.4195 & {$[0.001]$} \\
\hline 25 & -0.0053 & & -0.0090 & & 52.4509 & {$[0.001]$} \\
\hline 26 & 0.0004 & & 0.0151 & & 52.4510 & {$[0.002]$} \\
\hline 27 & 0.0266 & & 0.0232 & & 53.2452 & {$[0.002]$} \\
\hline 28 & -0.0406 & & -0.0587 & $*$ & 55.1052 & {$[0.002]$} \\
\hline 29 & -0.0775 & $* *$ & -0.0687 & $* *$ & 61.8709 & {$[0.000]$} \\
\hline 30 & -0.0257 & & -0.0076 & & 62.6158 & {$[0.000]$} \\
\hline
\end{tabular}

ACF for Bitcoinreturns

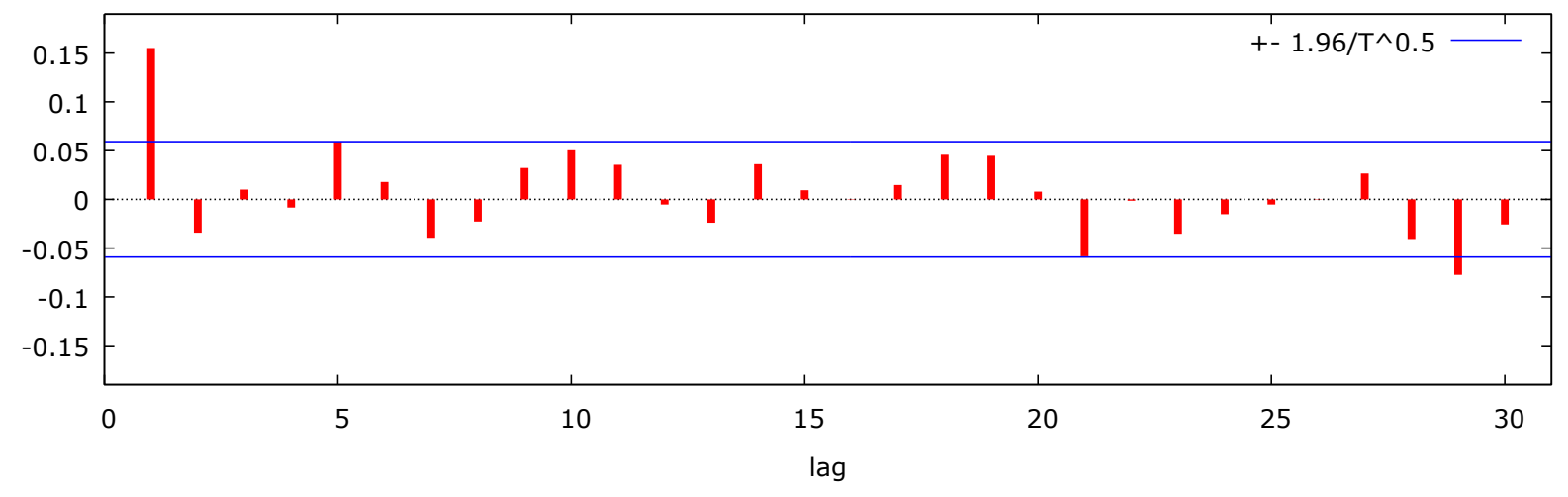

PACF for Bitcoinreturns

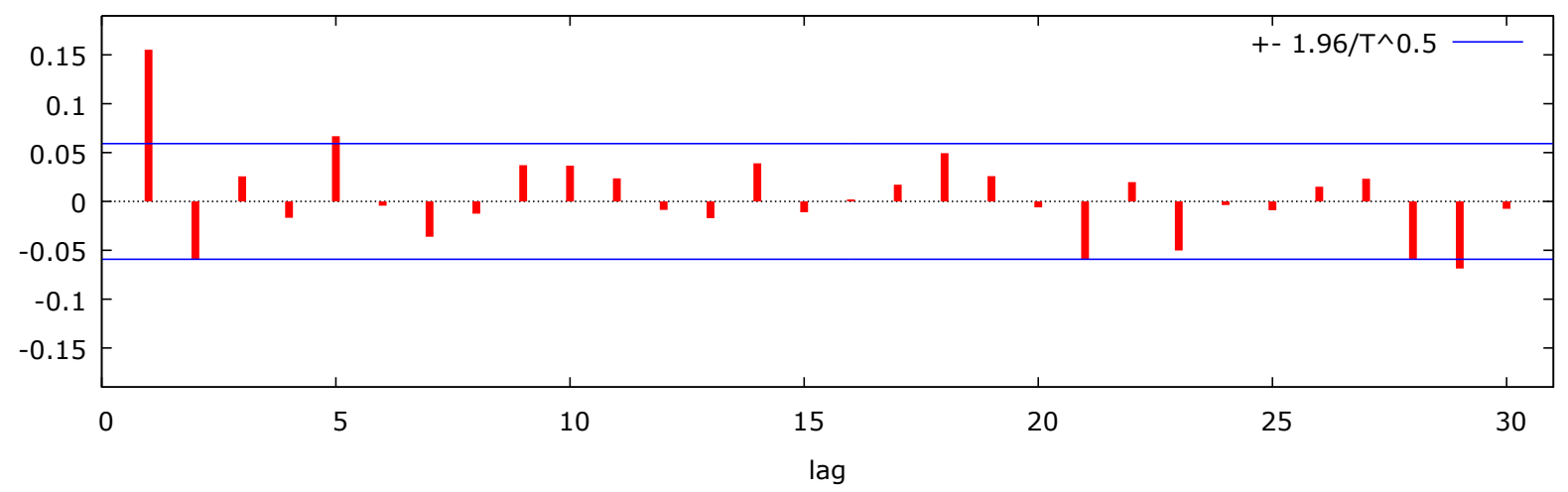

The ACF and PACF of Bitcoin returns were found to have similar patterns in both panels. However, there were differences in the pattern of ACF and PACF between the panels. There were significant autocorrelations and partial autocorrelations at the $1^{\text {st }}$ and $5^{\text {th }}$ lags in both panels. Further, there were significant autocorrelations and partial autocorrelations at the $8^{\text {th }}, 10^{\text {th }}, 17^{\text {th }}$, $20^{\text {th }}$, and $23^{\text {rd }}$ lags in panel A, while for panel B the autocorrelations and partial autocorrelations at the $21^{\text {st }}$ and $29^{\text {th }}$ lags were significant. This suggests that an ARMA model would be appropriate to model the series. 
The ARMA model estimates for Bitcoin returns are presented in the tables below.

Panel A (01/01/2013 - 31/12/2017):

Model 1: ARMA, using observations 2013-01-01:2017-12-31 ( $T=1826)$

Estimated using Kalman filter (exact $M L$ )

Dependent variable: Bitcoin_returns

Standard errors based on Hessian

$\begin{array}{lccccc} & \text { coefficient } & \text { std. error } & \mathrm{z} & \mathrm{p}-\mathrm{value} & \\ \text { const } & 0.00372382 & 0.00125158 & 2.975 & 0.0029 & * * * \\ \text { phi_1 } & 1.32096 & 0.0885326 & 14.92 & 2.42 \mathrm{e}-050 * * * \\ \text { phi_2 } & -1.32699 & 0.0686121 & -19.34 & 2.46 \mathrm{e}-083 * * * \\ \text { phi_3 } & 0.563438 & 0.0837538 & 6.727 & 1.73 \mathrm{e}-011 * * * \\ \text { theta_1 } & -1.14980 & 0.0972912 & -11.82 & 3.15 \mathrm{e}-032 & * * * \\ \text { theta_2 } & 1.11662 & 0.0768071 & 14.54 & 6.96 \mathrm{e}-048 * * * \\ \text { theta_3 } & -0.374737 & 0.0899452 & -4.166 & 3.10 \mathrm{e}-05 & * * *\end{array}$

$\begin{array}{lrlr}\text { Mean dependent var } & 0.003750 & \text { S.D. dependent var } & 0.041341 \\ \text { Mean of innovations } & 1.06 e-06 & \text { S.D. of innovations } & 0.039993 \\ \text { Log-likelihood } & 3286.861 & \text { Akaike criterion } & -6557.721 \\ \text { Schwarz criterion } & -6513.642 & \text { Hannan-Quinn } & -6541.462\end{array}$

Model 2: ARMA, using observations 2013-01-01:2017-12-31 ( $T=1826)$ Estimated using Kalman filter (exact $M L$ )

Dependent variable: Bitcoin_returns

Standard errors based on Hessian

$\begin{array}{lccccc} & \text { coefficient } & \text { std. error } & \text { z } & \text { p-value } & \\ \text { const } & 0.00372272 & 0.00125816 & 2.959 & 0.0031 & * * * \\ \text { phi_1 } & 0.376448 & 0.0853184 & 4.412 & 1.02 \mathrm{e}-05 & * * * \\ \text { phi_2 } & -0.0480315 & 0.0375122 & -1.280 & 0.2004 & \\ \text { phi_3 } & -0.715583 & 0.0426224 & -16.79 & 2.94 \mathrm{e}-063 & * * * \\ \text { phi_4 } & 0.566908 & 0.0773092 & 7.333 & 2.25 \mathrm{e}-013 & * * * \\ \text { theta_1 } & -0.195605 & 0.0936096 & -2.090 & 0.0367 & * * \\ \text { theta_2 } & -0.0205830 & 0.0456054 & -0.4513 & 0.6518 & \\ \text { theta_3 } & 0.724689 & 0.0494022 & 14.67 & 1.02 \mathrm{e}-048 * * * \\ \text { theta_4 } & -0.401966 & 0.0865966 & -4.642 & 3.45 \mathrm{e}-06 & * * *\end{array}$

Mean dependent var 0.003750 S.D. dependent var 0.041341

Mean of innovations $1.75 \mathrm{e}-06$ S.D. of innovations 0.039870

Log-likelihood $\quad 3292.382$ Akaike criterion -6564.763

Schwarz criterion -6509.664 Hannan-Quinn $\quad-6544.439$

Model 3: ARMA, using observations 2013-01-01:2017-12-31 ( $T=1826$ )

Estimated using Kalman filter (exact $M L$ )

Dependent variable: Bitcoinreturns

Standard errors based on Hessian

\begin{tabular}{llllll} 
& coefficient & std. error & \multicolumn{1}{c}{$\mathrm{z}$} & p-value & \\
const & 0.00372515 & 0.00124636 & 2.989 & 0.0028 & $* * *$ \\
phi_1 & -0.336384 & 0.114855 & -2.929 & 0.0034 & $* * *$ \\
phi_2 & -0.0847084 & 0.145945 & -0.5804 & 0.5616 & \\
phi_3 & -0.431283 & 0.0966263 & -4.463 & $8.07 \mathrm{e}-06$ & $* * *$ \\
phi_4 & -0.282620 & 0.103454 & -2.732 & 0.0063 & $* * *$ \\
phi_5 & 0.477338 & 0.101105 & 4.721 & $2.34 \mathrm{e}-06$ & $* * *$
\end{tabular}




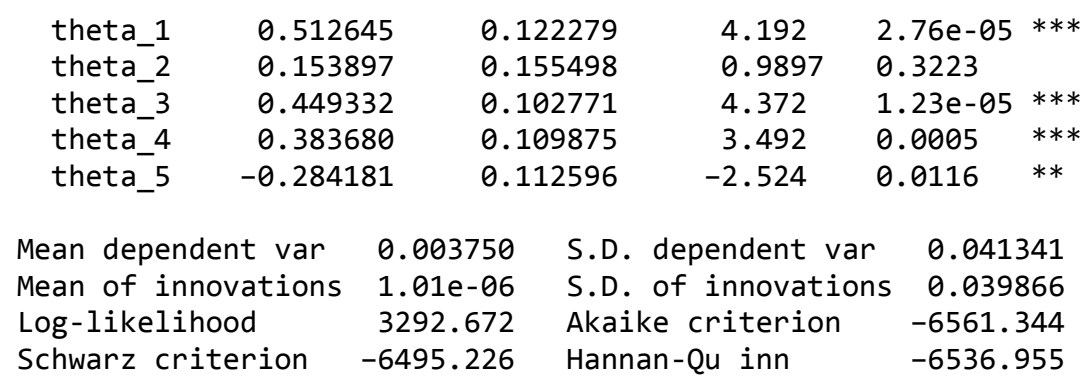

\section{Panel B (01/01/2015 - 31/12/2017):}

Model 1: ARMA, using observations 2015-01-01:2017-12-31 ( $T=1096)$

Estimated using Kalman filter (exact $M L$ )

Dependent variable: Bitcoin_returns

Standard errors based on Hessian

$\begin{array}{lccrll} & \text { coefficient } & \text { std. error } & \mathrm{z} & \text { p-value } \\ \text { const } & 0.00336879 & 0.00110242 & 3.056 & 0.0022 & * * * \\ \text { phi_1 } & -0.328622 & 0.172547 & -1.905 & 0.0568 & * \\ \text { theta_1 } & 0.495631 & 0.159695 & 3.104 & 0.0019\end{array} * * *$

$\begin{array}{lrlr}\text { Mean dependent var } & 0.003382 & \text { S.D. dependent var } & 0.032933 \\ \text { Mean of innovations } & -2.65 e-06 & \text { S.D. of innovations } & 0.032424 \\ \text { Log-likelihood } & 2202.865 & \text { Akaike criterion } & -4397.729 \\ \text { Schwarz criterion } & -4377.732 & \text { Hannan-Quinn } & -4390.163\end{array}$

The results of the ARMA model estimation for panel A indicate that the best models with respect to the AIC and $\operatorname{BIC}$ criteria were $\operatorname{ARMA}(4,4)$ and $\operatorname{ARMA}(3,3)$, respectively. On the other hand, the $\operatorname{ARMA}(5,5)$ model showed significant $\varphi_{5}$ and $\vartheta_{5}$ coefficients and had higher log-likelihood, but had higher AIC and BIC than the former models. All three of these models can be considered as appropriate models for Bitcoin returns.

On the other hand, the results of the ARMA model estimation for panel B indicate that the best model with respect to all three criteria was $\operatorname{ARMA}(1,1)$.

The results of the AR-GARCH models are presented in the tables below.

Panel A (01/01/2013 - 31/12/2017):

Model: $\operatorname{GARCH}(1,1)$ [Bollerslev] (Normal)*

Dependent variable: Bitcoin_returns

Sample: 2013-01-02 -- 2017-12-31 (

Conditional mean equation

\begin{tabular}{|c|c|c|c|c|c|}
\hline & coefficient & std. error & z & p-value & \\
\hline & a $9012157 a$ & a 990476328 & 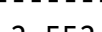 & & \\
\hline AR1 & 0.225169 & 0.0295263 & $\begin{array}{l}2.552 \\
7.626\end{array}$ & $2.42 \mathrm{e}-014$ & $* * *$ \\
\hline
\end{tabular}

Conditional variance equation

\begin{tabular}{llllll} 
& \multicolumn{1}{c}{ coefficient } & std. error & $\mathrm{z}$ & $\mathrm{p}-\mathrm{value}$ & \\
omega & $1.62198 \mathrm{e}-05$ & $6.31971 \mathrm{e}-06$ & 2.567 & 0.0103
\end{tabular}$* *$



beta
0.773459
0.0291573
26.53
$4.71 \mathrm{e}-155 * * *$
$\begin{array}{lrll}\text { Llik: } & 3833.41627 & \text { AIC: } & -7656.83254 \\ \text { BIC: } & -7629.28586 & \text { HQC: } & -7646.67107\end{array}$

Model: $\operatorname{GARCH}(1,1)$ [Bollerslev] (Normal)*

Dependent variable: Bitcoin_returns

Sample: 2013-01-06 - 2017-12-31 ( $T=1821)$, VCV method: Robust

Conditional mean equation

$\begin{array}{lccccc} & \text { coefficient } & \text { std. error } & \text { z } & \text { p-value } & \\ \text { const } & 0.00114861 & 0.000463102 & 2.480 & 0.0131 & * * \\ \text { AR1 } & 0.233063 & 0.0309269 & 7.536 & 4.85 \mathrm{e}-014 & * * * \\ \text { AR2 } & -0.0427224 & 0.0308597 & -1.384 & 0.1662 & \\ \text { AR3 } & 0.0382540 & 0.0307154 & 1.245 & 0.2130 & \\ \text { AR4 } & -0.0290588 & 0.0291487 & -0.9969 & 0.3188 & \\ \text { AR5 } & 0.0646978 & 0.0280800 & 2.304 & 0.0212 & * *\end{array}$

Conditional variance equation

\begin{tabular}{llllll} 
& coefficient & std. error & z & \multicolumn{1}{c}{ p-value } & \\
omega & $1.60188 \mathrm{e}-05$ & $6.27284 \mathrm{e}-06$ & 2.554 & 0.0107 & $* *$ \\
alpha & 0.267058 & 0.0441643 & 6.047 & $1.48 \mathrm{e}-09$ & $* * *$ \\
beta & 0.774968 & 0.0293130 & 26.44 & $5.05 \mathrm{e}-154$ & $* * *$
\end{tabular}

Llik: $\quad 3826.61805$ AIC: -7635.23610

BIC: $\quad-7585.67183$ HQC: -7616.95071

Model: $\operatorname{GARCH}(1,1)$ [Bollerslev] (Normal)*

Dependent variable: Bitcoin_returns

Sample: 2013-01-07 - 2017-12-31 ( $T=1820)$, VCV method: Robust

Conditional mean equation

\begin{tabular}{lccccc} 
& coefficient & std. error & $\mathrm{z}$ & \multicolumn{1}{c}{$\mathrm{p}$-value } & \\
const & 0.00110432 & 0.000461019 & 2.395 & 0.0166 & $* *$ \\
AR1 & 0.230633 & 0.0306677 & 7.520 & $5.46 \mathrm{e}-014$ & $* * *$ \\
AR2 & -0.0426079 & 0.0310242 & -1.373 & 0.1696 & \\
AR3 & 0.0364607 & 0.0305167 & 1.195 & 0.2322 & \\
AR4 & -0.0286436 & 0.0292709 & -0.9786 & 0.3278 & \\
AR5 & 0.0557621 & 0.0280686 & 1.987 & 0.0470 & $* *$ \\
AR6 & 0.0462937 & 0.0268039 & 1.727 & 0.0841 & $*$
\end{tabular}

Conditional variance equation

$\begin{array}{llllll} & \text { coefficient } & \text { std. error } & \text { z } & \text { p-value } & \\ \text { omega } & 1.61777 \mathrm{e}-05 & 6.25866 \mathrm{e}-06 & 2.585 & 0.0097 & * * * \\ \text { alpha } & 0.268739 & 0.0441863 & 6.082 & 1.19 \mathrm{e}-09 & * * * \\ \text { beta } & 0.773638 & 0.0291916 & 26.50 & 9.16 \mathrm{e}-155 & * * *\end{array}$

Llik: $\quad 3825.59347$ AIC: -7631.18694

BIC: $\quad-7576.12102$ HQC: -7610.87130

Model: $\operatorname{GARCH}(1,1)$ [Bollerslev] (Normal)* 
Dependent variable: Bitcoin_returns

Sample: 2013-01-08 - 2017-12-31 ( $T=1819)$, VCV method: Robust

Conditional mean equation

$\begin{array}{lccccc} & \text { coefficient } & \text { std. error } & z & \text { p-value } & \\ \text { const } & 0.00114563 & 0.000454421 & 2.521 & 0.0117 & * * \\ \text { AR1 } & 0.233691 & 0.0302732 & 7.719 & 1.17 \mathrm{e}-014 & * * * \\ \text { AR2 } & -0.0413357 & 0.0306436 & -1.349 & 0.1774 & \\ \text { AR3 } & 0.0342451 & 0.0303183 & 1.130 & 0.2587 & \\ \text { AR4 } & -0.0242986 & 0.0287539 & -0.8451 & 0.3981 & \\ \text { AR5 } & 0.0523958 & 0.0276314 & 1.896 & 0.0579 & * \\ \text { AR6 } & 0.0576161 & 0.0260215 & 2.214 & 0.0268 & * * \\ \text { AR7 } & -0.0525383 & 0.0269303 & -1.951 & 0.0511 & *\end{array}$

Conditional variance equation

$\begin{array}{llllll} & \text { coefficient } & \text { std. error } & \text { z } & \text { p-value } & \\ \text { omega } & 1.58601 \mathrm{e}-05 & 6.10038 \mathrm{e}-06 & 2.600 & 0.0093 & * * * \\ \text { alpha } & 0.275346 & 0.0454330 & 6.060 & 1.36 \mathrm{e}-09 & * * * \\ \text { beta } & 0.769257 & 0.0290645 & 26.47 & 2.31 \mathrm{e}-154 & * * *\end{array}$

$$
\text { Llik: } \quad 3825.23759 \text { AIC: }-7628.47518
$$

BIC: $\quad-7567.90871$ HQC : -7606.12959

Model: $\operatorname{GARCH}(1,1)$ [Bollerslev] (Normal)*

Dependent variable: Bitcoin_returns

Sample: 2013-01-23 -- 2017-12-31 ( $T=1804)$, VCV method: Robust

Conditional mean equation

$\begin{array}{lccccc} & \text { coefficient } & \text { std. error } & \text { z } & \text { p-value } & \\ \text { const } & 0.000950645 & 0.000446144 & 2.131 & 0.0331 & * * \\ \text { AR1 } & 0.233012 & 0.0300499 & 7.754 & 8.89 \mathrm{e}-015 & * * * \\ \text { AR2 } & -0.0373272 & 0.0301070 & -1.240 & 0.2150 & \\ \text { AR3 } & 0.0325942 & 0.0292789 & 1.113 & 0.2656 & \\ \text { AR4 } & -0.0341181 & 0.0287797 & -1.185 & 0.2358 & \\ \text { AR5 } & 0.0452607 & 0.0275861 & 1.641 & 0.1009 & \\ \text { AR6 } & 0.0597387 & 0.0256034 & 2.333 & 0.0196 & * * \\ \text { AR7 } & -0.0470114 & 0.0262174 & -1.793 & 0.0730 & * \\ \text { AR8 } & -0.0213515 & 0.0256324 & -0.8330 & 0.4049 & \\ \text { AR9 } & 0.00500548 & 0.0240879 & 0.2078 & 0.8354 & \\ \text { AR10 } & 0.0255735 & 0.0255341 & 1.002 & 0.3166 & \\ \text { AR11 } & 0.00477708 & 0.0247719 & 0.1928 & 0.8471 & \\ \text { AR12 } & -0.0271160 & 0.0245905 & -1.103 & 0.2702 & \\ \text { AR13 } & -0.00326454 & 0.0255385 & -0.1278 & 0.8983 & \\ \text { AR14 } & 0.0427626 & 0.0248259 & 1.723 & 0.0850 & * \\ \text { AR15 } & 0.0171878 & 0.0269989 & 0.6366 & 0.5244 & \\ \text { AR16 } & 0.00972889 & 0.0241233 & 0.4033 & 0.6867 & \\ \text { AR17 } & 0.0129611 & 0.0228686 & 0.5668 & 0.5709 & \\ \text { AR18 } & -0.0125906 & 0.0222389 & -0.5662 & 0.5713 & \\ \text { AR19 } & 0.0194808 & 0.0229565 & 0.8486 & 0.3961 & \\ \text { AR20 } & 0.0129829 & 0.0235798 & 0.5506 & 0.5819 & \\ \text { AR21 } & -0.0282592 & 0.0217867 & -1.297 & 0.1946 & \\ \text { AR22 } & 0.0395271 & 0.0225362 & 1.754 & 0.0794 & *\end{array}$

Conditional variance equation 


$\begin{array}{llllll} & \text { coefficient } & \text { std. error } & \text { z } & \text { p-value } & \\ \text { omega } & 1.54035 \mathrm{e}-05 & 5.85565 \mathrm{e}-06 & 2.631 & 0.0085 & * * * \\ \text { alpha } & 0.281951 & 0.0494783 & 5.698 & 1.21 \mathrm{e}-08 & * * * \\ \text { beta } & 0.764704 & 0.0313845 & 24.37 & 3.96 \mathrm{e}-131 & * * *\end{array}$

Llik: $\quad 3800.19093$ AIC: -7548.38185

BIC: $\quad-7405.44005$ HQC: -7495.62242

Panel B (01/01/2015 - 31/12/2017):

Model: $\operatorname{GARCH}(1,1)$ [Bollerslev] (Normal)*

Dependent variable: Bitcoin_returns

Sample: 2015-01-02 - 2017-12-31 (

Conditional mean equation

\begin{tabular}{|c|c|c|c|c|}
\hline & coefficient & std. error & z & p-value \\
\hline & a 091 & 0 & 2 & \\
\hline AR1 & 0.213178 & 0.0365509 & 5.832 & $5.46 e-09$ \\
\hline
\end{tabular}

Conditional variance equation

$\begin{array}{llllll} & \text { coefficient } & \text { std. error } & \text { z } & \text { p-value } & \\ \text { omega } & 1.08146 \mathrm{e}-05 & 4.71648 \mathrm{e}-06 & 2.293 & 0.0219 & * * \\ \text { alpha } & 0.218956 & 0.0416002 & 5.263 & 1.41 \mathrm{e}-07 & * * * \\ \text { beta } & 0.804679 & 0.0310790 & 25.89 & 8.32 \mathrm{e}-148 & * * *\end{array}$

Llik: 2495.66518 AIC: -4981.33037

BIC: $\quad-4956.33782$ HQC: -4971.87340

Model: $\operatorname{GARCH}(1,1)$ [Bollerslev] (Normal)*

Dependent variable: Bitcoin_returns

Sample: 2015-01-31 - 2017-12-31 (

Conditional mean equation

\begin{tabular}{|c|c|c|c|c|c|}
\hline & coefficient & std. error & z & p-value & \\
\hline & & & & & \\
\hline const & 0.00155461 & 0.000483178 & 3.217 & 0.0013 & \\
\hline AR1 & 0.215863 & 0.0356427 & 6.056 & $1.39 e-09$ & $* *$ \\
\hline AR2 & -0.0469729 & 0.0342711 & -1.371 & 0.1705 & \\
\hline AR3 & 0.00980338 & 0.0338962 & 0.2892 & 0.7724 & \\
\hline AR4 & -0.0402046 & 0.0344394 & -1.167 & 0.2430 & \\
\hline AR5 & 0.0301559 & 0.0337880 & 0.8925 & 0.3721 & \\
\hline AR6 & 0.0609462 & 0.0318737 & 1.912 & 0.0559 & * \\
\hline AR7 & -0.0560488 & 0.0314022 & -1.785 & 0.0743 & $*$ \\
\hline AR8 & -0.0549245 & 0.0311780 & -1.762 & 0.0781 & $*$ \\
\hline AR9 & 0.00208956 & 0.0305568 & 0.06838 & 0.9455 & \\
\hline AR10 & 0.0268740 & 0.0290504 & 0.9251 & 0.3549 & \\
\hline AR11 & 0.00951606 & 0.0302008 & 0.3151 & 0.7527 & \\
\hline AR12 & -0.0240190 & 0.0305697 & -0.7857 & 0.4320 & \\
\hline AR13 & -0.00864688 & 0.0313523 & -0.2758 & 0.7827 & \\
\hline AR14 & 0.0531280 & 0.0286303 & 1.856 & 0.0635 & $*$ \\
\hline AR15 & -0.00568987 & 0.0326670 & -0.1742 & 0.8617 & \\
\hline AR16 & -0.00470319 & 0.0300438 & -0.1565 & 0.8756 & \\
\hline AR17 & 0.000130166 & 0.0254348 & 0.005118 & 0.9959 & \\
\hline
\end{tabular}




\begin{tabular}{|c|c|c|c|c|c|}
\hline AR18 & 0.0143437 & 0.0253718 & 0.5653 & 0.5718 & \\
\hline AR19 & 0.0128481 & 0.0267110 & 0.4810 & 0.6305 & \\
\hline AR20 & -0.0390407 & 0.0278419 & -1.402 & 0.1608 & \\
\hline AR21 & -0.0427248 & 0.0257570 & -1.659 & 0.0972 & \\
\hline AR22 & 0.0600753 & 0.0278995 & 2.153 & 0.0313 & \\
\hline AR23 & 0.00673543 & 0.0274744 & 0.2452 & 0.8063 & \\
\hline AR24 & -0.000125798 & 0.0277502 & -0.004533 & 0.9964 & \\
\hline AR25 & -0.00571637 & 0.0277826 & -0.2058 & 0.8370 & \\
\hline AR26 & 0.0300101 & 0.0285684 & 1.050 & 0.2935 & \\
\hline AR27 & 0.0196746 & 0.0333506 & 0.5899 & 0.5552 & \\
\hline AR28 & 0.000961953 & 0.0383573 & 0.02508 & 0.9800 & \\
\hline AR29 & -0.0765444 & 0.0334690 & -2.287 & 0.0222 & \\
\hline AR30 & -0.0237276 & 0.0346463 & -0.6849 & 0.4934 & \\
\hline \multicolumn{6}{|c|}{ Conditional variance equation } \\
\hline & coefficient & std. error & z & \multicolumn{2}{|l|}{ p-value } \\
\hline \multicolumn{6}{|l|}{-----} \\
\hline omega & $1.14310 \mathrm{e}-05$ & $4.85148 \mathrm{e}-06$ & 2.356 & \multicolumn{2}{|l|}{0.0185} \\
\hline alpha & 0.264934 & 0.0515745 & 5.137 & $2.79 e-07$ & $* * *$ \\
\hline beta & 0.769047 & 0.0346654 & 22.18 & $4.81 \mathrm{e}-109$ & $* * *$ \\
\hline Llik: & 2484.22710 & AIC: & .45419 & & \\
\hline BIC: & -4731.41746 & $\mathrm{HQC}:$ & 5.40808 & & \\
\hline
\end{tabular}

The results of the AR-GARCH models show non-stationarity of the conditional variance (as all models have $\alpha_{1}+\beta_{1}>1$ ). The results also show significant AR terms at the $1^{\text {st }}, 5^{\text {th }}, 6^{\text {th }}, 7^{\text {th }}, 13^{\text {th }}$, and $22^{\text {nd }}$ lags. According to the AIC and BIC criteria, the best model was AR(1)-GARCH(1, 1).

\section{Discussion}

The results of the study suggest that Bitcoin returns has non-stationary conditional variance. This may be interpreted as a speculative bubble, as some noted economists, including Nobel laureates Paul Krugman, Robert J. Shiller, Joseph Stiglitz, Richard Thaler, James Heckman, Thomas Sargent, Angus Deaton, and Oliver Hart, had observed. In fact, Bitcoin prices crashed drastically in late 2017, conforming this observation. The results are related with those presented by Katsiampa (2017).

Comparing the results of Panel A and Panel B, some similarities and differences are evident. The first and fifth ACFs and PACFs were significant for both panels; on the other hand, the tenth, fifteenth, and twentieth ACFs and PACFs were significant in Panel A, they were not significant in Panel B, while the twenty-ninth ACFs and PACFs were significant in Panel B, though they were not significant in Panel A. This is reflected in the ARMA results; for Panel A, $\operatorname{ARMA}(3,3)$, $\operatorname{ARMA}(4,4)$, and $\operatorname{ARMA}(5,5)$ were the optimal models, while for Panel B, only ARMA $(1,1)$ was the best model. Both of these results suggest that that Bitcoin returns have a simpler lag structure in Panel B than in Panel A. This provides some evidence of change in the time series properties of Bitcoin returns post-2015.

The AR-GARCH model results, however, indicate that the non-stationarity of Bitcoin returns conditional variance persists. Thus, Bitcoin price volatility does not show evidence of stabilising, as suggested by Bouoiyour and Selmi (2016). 
There are several limitations inherent in the study. The study has used data from only one Bitcoin exchange, and the study period was limited to five years. Also, the study did not consider macroeconomic factors affecting Bitcoin prices, including particularly exchange rates of some of the important currencies, interest rates, inflation, gold prices, and oil prices. Multivariate econometric models including such factors may better explain the instability of Bitcoin returns.

\section{References}

Bouoiyour, J. and Selmi, R. (2016), "Bitcoin: A beginning of a new phase?" Economics Bulletin 36(3), 1430-1440.

Cheah, E.T. and Fry, J. (2015), "Speculative bubbles in Bitcoin markets? An empirical investigation into the fundamental value of Bitcoin," Economics Letters 130, 32-36.

Chu, J., Stephen, C., Saralees, N., and Joerg, O. (2017), "GARCH Modelling of Cryptocurrencies," Journal of Risk and Financial Management 10(4), 1-15.

Katsiampa, P. (2017), "Volatility estimation for Bitcoin: A comparison of GARCH models," Working Paper, Sheffield Hallam University Research Archive.

Stavroyiannis, S. and Babalos, V. (2017), "Dynamic properties of the Bitcoin and the US market," SSRN Working Papers, available at: https://ssrn.com/abstract=2966998.

Zheng, H. (2018), "A systematic review of forecasting the cryptocurrency volatility using GARCH model," Coinmonks, available at: https://medium.com/coinmonks/a-systematic-review-offorecasting-the-cryptocurrency-volatility-using-garch-model-d1855c9779c 